\title{
Article
}

\section{Human Genetics Society of Australasia Position Statement: Predictive and Presymptomatic Genetic Testing in Adults and Children}

\author{
Danya F. Vears ${ }^{1,2}$, Samantha Ayres ${ }^{3,4,5}$, Jackie Boyle ${ }^{6}$, Julia Mansour ${ }^{7}$, Ainsley J. Newson ${ }^{8}$ and on behalf of the Education, \\ Ethics and Social Issues Committee of the Human Genetics Society of Australasia \\ ${ }^{1}$ Melbourne Law School, University of Melbourne, Melbourne, VIC, Australia, ${ }^{2}$ Biomedical Ethics Research Group, Murdoch Children's Research Institute, \\ Melbourne, VIC, Australia, ${ }^{3}$ Victorian Clinical Genetics Services, Murdoch Children's Research Institute, Melbourne, VIC, Australia, ${ }^{4}$ Melbourne Genomics Health \\ Alliance, Melbourne, VIC, Australia, ${ }^{5}$ Australian Genomics Health Alliance, Melbourne, VIC, Australia, ${ }^{6} \mathrm{Hunter}$ Genetics, Waratah, NSW, Australia, ${ }^{7}$ Tasmanian \\ Clinical Genetics Service, Royal Hobart Hospital, Hobart, TAS, Australia and ${ }^{8}$ Faculty of Medicine and Health, Sydney School of Public Health, Sydney Health \\ Ethics, The University of Sydney, Sydney, NSW, Australia
}

\begin{abstract}
In 2020, the Human Genetics Society of Australasia released its Position Statement on Predictive and Presymptomatic Genetic Testing in Adults and Children. This Position Statement synthesizes the major practical, psychosocial and ethical considerations associated with presymptomatic and predictive genetic testing in adults who have the capacity to make a decision, children and young people who lack capacity and adults living with reduced or fluctuating capacity. Recommendations include that predictive testing in adults, young people and children should only be offered with pretest genetic counseling and the option of posttest genetic counseling. An individual considering (for themselves or on behalf of another) whether to have a predictive test should also be supported to allow them to make an autonomous and informed decision. Predictive testing should only be offered to children and young people for conditions where there is likely to be a direct medical benefit to them through surveillance, use of prevention strategies or other medical interventions in the immediate future. Where symptoms are likely to develop in childhood, in the absence of options to implement surveillance or risk reduction measures, genetic health professionals and parents/guardians should discuss whether undertaking predictive testing is the best course of action for the child and the family as a whole. Where symptoms are likely to develop in adulthood, the default position should be to postpone predictive testing until the young person achieves the capacity to make their own autonomous and informed decision.
\end{abstract}

Keywords: adults; Australia; New Zealand; children; genetic testing; predictive testing; young people

(Received 30 April 2020; accepted 30 April 2020)

Genetic/genomic testing is becoming more widely available within clinical genetic and mainstream medical services. Depending on whether the test is a diagnostic, screening, presymptomatic or predictive test, a range of potential consequences should be considered prior to testing.

This Position Statement from the Human Genetics Society of Australasia (HGSA) presents the major practical, psychosocial and ethical considerations associated with presymptomatic and predictive genetic testing in adults who have the capacity to make a decision, children and young people who lack capacity and adults living with reduced or fluctuating cognitive capacity. The terms

Author for correspondence: Danya F. Vears, Email: dvears@unimelb.edu.au Cite this article: Vears DF, Ayres S, Boyle J, Mansour J, and Newson AJ on behalf of the Education, Ethics and Social Issues Committee of the Human Genetics Society of Australasia. (2020) Human Genetics Society of Australasia Position Statement: Predictive and Presymptomatic Genetic Testing in Adults and Children. Twin Research and Human Genetics 23: 184-189, https://doi.org/10.1017/thg.2020.51 'presymptomatic testing' and 'predictive testing' are often used interchangeably. This statement will use the term 'predictive testing' (defined in Table 1) to encompass both presymptomatic and predictive testing.

This statement also provides guidelines for health professionals who work with individuals and families seeking predictive genetic testing and laboratory staff conducting the tests.

Figure 1 presents a flow chart summarizing the differences between diagnostic and predictive testing and the associated considerations. This Position Statement contains practice guidelines that will apply to predictive tests undertaken by accredited laboratories for

- adults who have the capacity to make a decision,

- children and young people who lack capacity and

- adults with reduced capacity.

This guidance applies whether testing is offered in a clinical setting by commercial providers (either clinically mediated or 'direct

(c) The Author(s) 2020. This is an Open Access article, distributed under the terms of the Creative Commons Attribution-NonCommercial-NoDerivatives licence (http://creativecommons. org/licenses/by-nc-nd/4.0/), which permits non-commercial re-use, distribution, and reproduction in any medium, provided the original work is unaltered and is properly cited. The written permission of Cambridge University Press must be obtained for commercial re-use or in order to create a derivative work. 
Table 1. Definitions of terms

\begin{tabular}{|c|c|}
\hline Pathogenic variant & $\begin{array}{l}\text { A genetic variation (sometimes known as a mutation) which is proven, or strongly predicted to, cause, } \\
\text { or predispose to, a given condition }\end{array}$ \\
\hline Diagnostic genetic test & $\begin{array}{l}\text { A test to identify the genetic cause of a condition in an individual with clinical signs and symptoms } \\
\text { associated with the condition }\end{array}$ \\
\hline Screening genetic test & $\begin{array}{l}\text { A test offered to a population defined by a set of characteristics, such as age or ethnicity, who may or may not have a } \\
\text { family history of the screened condition(s) }\end{array}$ \\
\hline $\begin{array}{l}\text { Presymptomatic and predictive } \\
\text { genetic test }\end{array}$ & $\begin{array}{l}\text { A genetic test performed when there is a family history of a particular condition and the causative pathogenic variant is } \\
\text { known. In most instances, the individual being tested does not have clinical features or a diagnosis of the condition }\end{array}$ \\
\hline Presymptomatic genetic test & $\begin{array}{l}\text { The presence of a pathogenic variant indicates that the individual tested is almost certain to develop the condition } \\
\text { during their lifetime }\end{array}$ \\
\hline Predictive genetic test & $\begin{array}{l}\text { The presence of a pathogenic variant indicates that the individual tested has an increased chance of developing the } \\
\text { condition. However, not everyone with the pathogenic variant will develop the condition }\end{array}$ \\
\hline Children and young people & $\begin{array}{l}\text { For the purposes of this document, the term 'child' refers to those under } 18 \text { years of age, } 18 \text { years being the legal age of } \\
\text { majority throughout Australasia. There is also growing use of the term 'young people', which encompasses individuals } \\
\text { from } 10 \text { to } 24 \text { years of age, recognizing that there is a continuum of developing capacity and that young people should } \\
\text { be included in decision-making regarding their health in an age-appropriate way from quite early in life }\end{array}$ \\
\hline
\end{tabular}

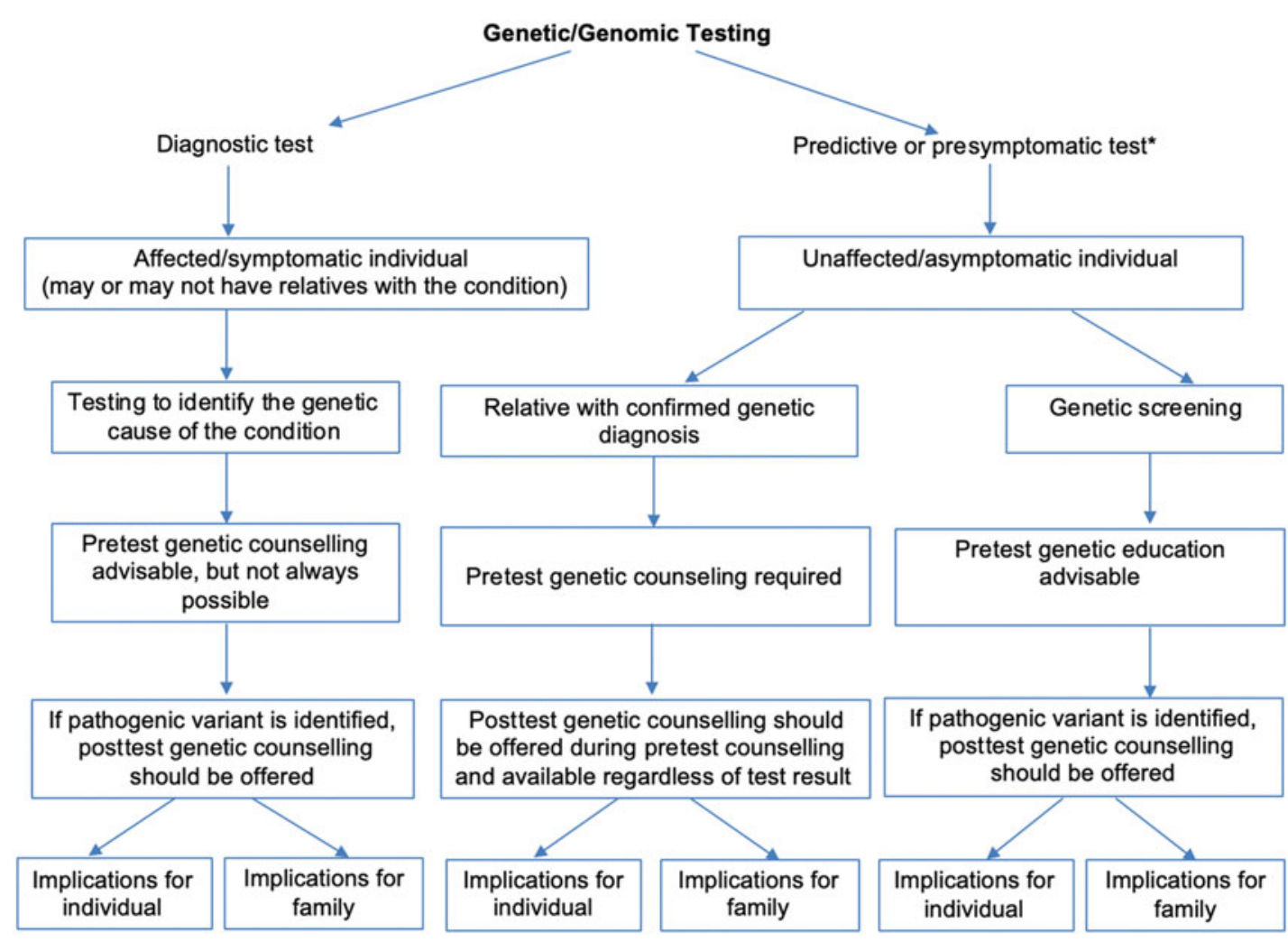

* Refer to definitions in Table 1

Fig. 1. Flow chart of the differences between diagnostic and predictive testing and the associated considerations.

to consumer'1) or under a research protocol. It does not apply to community or population screening for genetic conditions, carrier screening/testing or identification of incidental/unsolicited findings in the course of diagnostic testing.

${ }^{1}$ More information about online DNA testing can be found in the HGSA Online DNA Position Statement (2019 PS01, https://www.hgsa.org.au/documents/item/18).
Figure 2 presents a flow chart summarizing the decision-making pathway for predictive testing.

\section{General Considerations in Predictive Testing}

There are a number of considerations when offering predictive testing: 


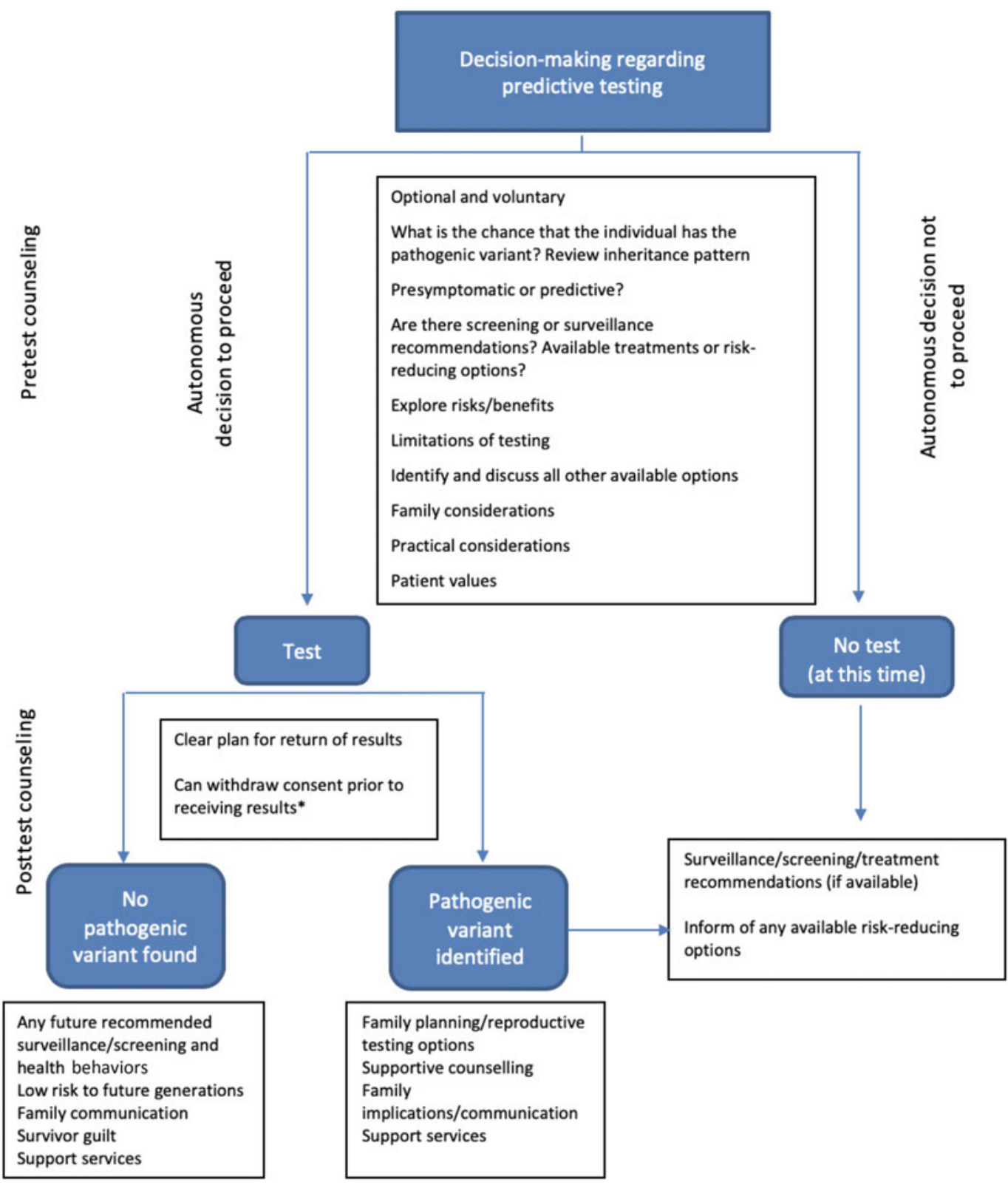

*If a report has been generated at the time of withdrawal of consent, the result may still need to be lodged in the patient's file, marked not for communication to patient.

Fig. 2. Flow chart of the decision-making pathway for predictive testing.

- Is there a known genetic diagnosis of the condition in the family?

- What is the predictive value of the relevant variant? Does it confer an increased risk (i.e., a predisposition), and if so, what is the degree of risk? Or does it confer a prediction of future health status?

- What is known about the likely age of developing the condition, the natural history of the condition and the likely symptoms?

- Are surveillance options, preventative treatments or symptombased treatments available? If so, at what age should they commence? Would they change in the presence of a positive result?
- Are there any psychosocial benefits associated with understanding personal risk of developing the particular condition? Could this information also be of value to other family members?

\section{How Should the Test Be Provided?}

Predictive testing is available when the specific pathogenic variant causing the genetic condition has been identified in an affected family member.

Predictive testing should only be offered with pretest genetic counseling, and the option or availability of posttest result genetic 
counseling, if required. The individual may want to bring an appropriate support person, such as a family member or friend to the appointment. Genetic counseling assists the individual and family to make informed decisions that align with their perceived interests and personal values, based on an understanding of the range of alternative options, the testing process and the possible implications of predictive genetic testing.

In most circumstances, genetic counseling for predictive testing is provided by genetic counselors. Most genetic counselors work in association with multidisciplinary teams, which may include clinical geneticists, laboratory scientists and medical specialists from a range of other disciplines including oncology, cardiology, neurology and pediatrics. All professionals offering pretest genetic counseling for predictive testing must be knowledgeable about the genetic condition for which testing is being considered and possible issues associated with accuracy and interpretation of the laboratory test.

Molecular testing should have a program of quality control and audit similar to the standards recommended by the National Pathology Accreditation Advisory Council Standards (2017). Laboratories performing predictive tests should develop protocols, in consultation with those delivering the clinical service, governing the conditions under which samples will be accepted for testing. This may include defining the circumstances in which two samples are to be provided for predictive testing to serve as an internal quality control, and the preference to use a positive familial control.

\section{Counseling Considerations}

Predictive genetic testing should not be performed without the consenting individual's (or their parent/guardian's) knowledge of, and participation in, the counseling process. When considering whether to continue with a predictive test for a known familial pathogenic variant, every effort should be made to assist the individual or their parent/guardian to make an autonomous and informed decision. This involves considering their capacity to make the best decision for themselves based on their perceived interests and personal values. It is imperative that the individual considering testing is aware that predictive testing is voluntary, and that a decision to proceed should be made without coercion or undue influence, in a time frame that suits them.

An individual's reasons for seeking testing and their expectations of the test should be explored. Even if the individual seems to have made a decision to proceed with testing, it is important that there is an opportunity to engage with the decision being made. Appropriate information, counseling and support are needed in both pre- and posttests. Interpreters should be used as required.

This information should include (but is not limited to) the following points:

- Risks and benefits of proceeding (or not) with testing, including the potential emotional impact on the individual and other family members and the potential impact on family relationships.

- Possible outcomes of the test (typically whether a pathogenic variant is identified, or no pathogenic variant is found) and the potential implications of those outcomes.

- All available alternatives to predictive testing, for example, if an individual does not proceed with predictive testing, are screening or preventative measures available?

- The inheritance pattern and possible implications for other family members, for example, testing an individual at $25 \%$ risk for a dominant disorder may reveal that an asymptomatic parent has the pathogenic variant and will develop the disease. Although this fact should not override an individual's access to testing, it should be considered and discussed in counseling before testing. Efforts should be made to involve other at-risk relative(s) in counseling, where applicable.

- Possible reproductive testing options, such as prenatal diagnosis, donor gametes or preimplantation genetic testing (sometimes referred to as preimplantation genetic diagnosis).

- Practical information, such as sample requirements, out-ofpocket costs (if applicable), test limitations, that only the familial pathogenic variant will be tested, expected turn-around-time, and that, in some instances, testing may reveal nonpaternity/ nonmaternity.

- For some conditions, pretest physical and psychological assessment is recommended (e.g., Huntington disease).

- A positive test result may adversely affect the ability to obtain or upgrade individually risk-rated insurance policies (such as life or income protection insurance). Once tested, applicants for insurance have a duty to disclose their genetic test result when applying for a new or upgraded insurance policy. ${ }^{2}$

- The possibility of withdrawal from the testing process at any time, including after the test has been performed but prior to receiving results.

- That the same procedure for return of results will be followed, regardless of the nature of the result.

In some cases, the testing process may require more than one appointment. For some conditions, the process of obtaining consent and sample collection may take place at separate appointments. If the 'at-risk' individual or their partner is pregnant and they are considering prenatal testing, the consent and sample collection appointments may be combined.

Occasionally, to facilitate testing in an individual, additional blood/saliva samples, clinical examination or access to medical/ genetic records of other family members may be required. In general, the individual requesting testing would approach the relevant family members who need to be involved in the testing process. This should be undertaken with sensitivity, recognizing that some family members may not wish to have genetic testing or to even discuss the condition in the family. In addition, some family structures or cultures might require further consultation and wider consent prior to testing.

\section{Confidentiality}

The individual considering predictive testing should be informed about how their personal information is stored and accessed. Every effort should be made by the service offering predictive testing to maintain the individual's privacy and confidentiality. Any documentation related to the individual's decision-making process and/or the outcome of predictive testing (i.e., test results and relevant correspondence) should be stored appropriately and only accessed with the consent of the individual or as required by law. For more information on confidentiality, please see the

\footnotetext{
${ }^{2} \mathrm{~A}$ moratorium was put in place by the Financial Services Council (FSC Standard No. 11 - Moratorium on Genetic Tests in Life Insurance), effective from July 1, 2019 and due to expire on June 30,2024 , which enables individuals to access up to $\$ 500,000$ of life insurance cover without being required to disclose a genetic test result (https:// www.fsc.org.au/policy/life-insurance). For more information on insurance, please see the HGSA's Position Statement on Genetic Testing and Personal Insurance Products in Australia (2018PS01, https://www.hgsa.org.au/documents/item/20).
} 
HGSA's Position Statement on Protection of Human Genetic Information held in the Clinical Setting (205GD01, https://www. hgsa.org.au/documents/item/6089).

\section{Return of Results}

A plan for result return should be arranged at the time of agreeing to testing. This should include discussion about an appropriate support person (family or friend) to bring to the appointment and a possible support network (family, friends, minister of religion, community groups, health or welfare professionals) that may be available if required once the result is known. The need for posttest counseling should be discussed with the participant prior to testing, bearing in mind that posttest counseling may be equally important whether or not a pathogenic variant is identified.

If at all possible, the results should be communicated in-person by the individual who provided pretest counseling (or one of the members of the team providing testing). Some individuals may ask for their result to be given by someone outside the testing team (e.g., the family doctor or another health professional). This should be agreed to, if appropriate, provided that the usual posttest information, support, counseling and follow-up are provided by that health professional, or jointly with the team.

Referral for further assistance from another health professional (e.g., psychiatrist, family therapist, social worker or psychologist) or support organization may be appropriate for some people, either prior to testing and/or in the posttest period. The timetable for follow-up, which should be agreed upon prior to testing, should be reviewed at the result appointment, and arrangements made for the first follow-up contact by the appropriate health professional, if required.

\section{Other Important Points to Consider}

There are specific genetic counseling guidelines for certain adultonset conditions for which there are no available treatments (e.g., Huntington's disease). For certain populations (e.g., Ashkenazi Jewish) testing for a single, known familial variant is not recommended. Broader testing to include all known pathogenic, founder variants or sequencing of the gene should be offered when individuals of certain ethnicities are seeking predictive testing.

Special care should be taken if a predictive test is requested by someone who appears to be affected by the condition for which testing is sought. This may reflect the psychological defense mechanisms of the individual (e.g., denial), which can be important for maintaining wellbeing and social functioning. A clinical opinion may be the next appropriate step after counseling or discussion, rather than predictive testing. If appropriate, diagnostic testing can follow clinical assessment to confirm a diagnosis. In some cases, if the health professional thinks the individual may react adversely to a diagnosis at that time, it may be appropriate to suggest deferring predictive testing, even if the diagnosis is not confirmed. Alternatively, predictive testing may proceed, noting that it will confirm a diagnosis in the patient. There are some advantages in taking this approach for patients who are unaware of their symptoms, including the ability to receive genetic counseling about the condition, and an opportunity to consider how they will incorporate a positive result into their lives. Provided the individual requesting predictive testing accepts that the test may confirm that their current symptoms are associated with the family condition, predictive testing may still be offered.

\section{Additional Considerations for Children and Young People}

\section{Assessing Capacity to Make Medical/Health Decisions}

Young people can be 'immature' (i.e., do not have the cognitive capacity and psychosocial maturity to make the decision to have a predictive test) or 'mature' (i.e., have such a capacity). Regarding the latter:

- In South Australia, specific legislation exists to allow young people aged 16 years and over to be treated as adults for the purposes of consenting to medical treatment.

- In all other states and territories, any young person under the age of 18 years may be deemed to have capacity to make a decision for themselves if they have 'sufficient understanding and intelligence' to enable a full understanding of the particular medical intervention being proposed. This type of capacity has been termed 'Gillick' competence. ${ }^{3}$ The Gillick competence rule provides a legal tool for making an assessment of whether the child is competent to make their own health decisions.

For people aged under 18 years who do not have legal capacity, usually the person(s) with legal responsibility for them will make the decision on their behalf. This decision should be one that is made in the best interests of the child, although it is noted that what constitutes 'best interests' is the subject of ongoing ethical debate. Psychological assessment may be required to determine an individual's cognitive and psychosocial maturity and their ability to understand genetic concepts and make an informed decision. Individuals need to appreciate that the decisions they make might have long-term consequences for psychological health, social circumstances, relationships, employment and ability to obtain certain insurance products. However, even young people without the capacity to make a decision can, and should, be included in the decision-making process. The appropriate level of inclusion in the decision will depend on their age, experiences and level of maturity.

In the event of a dispute between the young person and their parents regarding predictive genetic testing, the health professional should act as an advocate for the young person. However, resolution of such a dispute should recognize that the young person is part of a family, with counseling focusing on the family and young person together and separately.

\section{Circumstances in Which Testing May Be Appropriate in Young People or Children}

There are a range of contexts in which predictive testing in children might be considered. Whether testing is appropriate depends on a range of factors, such as the age at which symptoms of the condition are likely to start and whether treatment, surveillance or preventative measures can be taken in response to the knowledge of genetic status. For simplicity, these contexts can be broken down into the following broad categories:

Category 1: Childhood onset, actionable [e.g., familial adenomatous polyposis (FAP)]

Category 2: Childhood onset, nonactionable (e.g., retinitis pigmentosa)

Category 3: Adult onset, actionable (e.g., hereditary breast and ovarian cancer)

Category 4: Adult onset, nonactionable (e.g., Huntington disease).

${ }^{3}$ For more information, see Griffith (2016). 
Systematic reviews of the literature have shown a lack of research studies on the psychosocial impact of predictive testing in children and young people. Findings from the research that does exist suggest that testing has been most commonly undertaken for FAP and hereditary cardiac disease risk (Wakefield et al., 2016). While several studies showed nonsignificant increases in depression scores following testing, the majority concluded that shortterm adverse psychological outcomes of predictive testing, such as anxiety and depression after testing, were infrequent. There is, however, some evidence to suggest that children with a parent affected by the condition, and those who test positive, may be more at risk of adverse outcomes.

For Category 1 (i.e., predictive testing in children and young people where the condition is likely to present in childhood and there is some action that can be taken to treat, monitor or prevent the condition), testing does not have to be delayed until the child is able to make their own decision about whether they want to know their genetic status. Testing would have direct benefit for the child or young person and so would likely be in their best interests. Where possible, it is still valuable to include the child or young person in the discussion about the testing process. In rare circumstances where parents refuse the offer of a test, genetic services should work with families, striving to maintain openness and trust. If it is felt that testing is strongly indicated but still refused, the service could refer the case to a clinical ethics support service (if available) or, if refusal is intractable and held over a period of time, to the service's legal team.

For Category 2, where symptoms are likely to develop in childhood, yet there are no treatment or preventative strategies available (i.e., no direct medical benefit), genetic health professionals and parents/legal guardians should discuss whether undertaking predictive testing is the best course of action for the child and the family as a whole. Considerations could include the potential for nonmedical benefits, such as future planning for the child, and the family's ability to cope with uncertainty versus potentially negative information relating to the child's future health status.

For Categories 3 and 4 (i.e., predictive testing for adult-onset conditions in children or young people who do not have the current capacity to make their own testing decisions), the default position should always be to postpone testing until the child or young person achieves this capacity. This position values preserving the child or young person's future autonomy to make their own choice whether they wish to know their genetic risk of the condition.

However, there may be situations where it is considered appropriate for a child or young person to undergo such predictive testing, even when the young person is not able to fully engage with the testing process. In such rare circumstances, a decision may be reached between the health professionals and the family that it is appropriate to perform testing in the young person because it is the best option to support the wellbeing of the child or young person through constructive family dynamics. When such requests arise, they should be discussed with other members of the genetics service or a clinical ethics committee (where available). For clarity, this section of the Position Statement should not be taken as HGSA endorsement of routine predictive testing for adult-onset conditions in young people who cannot consent.

Counseling should be provided using language that can be best understood by the child or young person and their parents. The child or young person should be given the option to be seen alone for at least part of each genetic counseling session. Follow-up counseling should be available from appropriate professionals. Parents/ guardians should be encouraged to prioritize the outcome of predictive testing in terms of the benefit to the individual rather than in terms of the benefit to others.

Whether or not predictive testing takes place, parents should be encouraged to foster an awareness in the child or young person of the genetic condition in the family and its implications. This enables the child or young person to be raised with this knowledge. Being able to discuss this information within the family, at different stages of maturity, will ultimately enable the child or young person to have a better understanding of the outcome of testing (if performed in childhood) or to make a more informed choice about predictive genetic testing once they are older.

\section{Adults With Reduced Capacity to Give Informed Consent}

People with a reduced cognitive capacity, such as adults living with intellectual disability, require special consideration. Regardless of the individual's capacity to provide informed consent, information needs to be provided in a manner appropriate to their cognitive ability, and efforts should be made to sensitively explore their understanding. If the individual does not have capacity, they should still be actively included in the counseling process along with their substitute decision-maker. A support person (carer, legal guardian, family member) should be present if possible and appropriate. Reasons for being offered the test should be explored to ensure that the individual with reduced capacity understands and is not being coerced or unduly influenced into undergoing testing. Testing may be offered to help plan medical and care needs of the individual being offered testing. There may also be situations where it is appropriate that testing is offered to benefit other family members. When this occurs, the decision should prioritize the wellbeing and interests of the individual being tested.

Acknowledgments. The authors thank the additional members of the Education, Ethics and Social Issues Committee of the HGSA: Aideen McInerney-Leo, Amanda Samanek, Amy Nisselle, Kate Dunlop, Bronwyn Terrill, Michael Gabbett and Jacqueline Savard. This statement was reviewed and approved by the HGSA Council in April 2020.

Financial Support. Danya Vears acknowledges the infrastructure funding received from the Victorian State Government through the Operational Infrastructure Support (OIS) Program.

Conflict of Interest. None.

Ethical Standards. Not applicable.

\section{References}

Griffith, R. (2016). What is Gillick competence? Human Vaccines \& Immunotherapeutics, 12, 244-247.

National Pathology Accreditation Advisory Council. (2017). Requirements for Human Medical Genome Testing Utilising Massively Parallel Sequencing Technologies. Australian Government Department of Health. https://www1.health.gov.au/internet/main/publishing.nsf/Content/healthnpaac-publication.htm

Wakefield, C.E., Hanlon, L.V., Tucker, K.M., Patenaude, A.F., Signorelli, C., McLoone, J.K., \& Cohn, R.J. (2016). The psychological impact of genetic information on children: A systematic review. Genetics in Medicine, 18, $755-762$. 